\title{
TriAG:Answering SPARQL Queries Accelerated by GPU
}

\author{
Jinhui Pang ${ }^{1}$, Shujun Wang ${ }^{2+}$, Jie Jiao ${ }^{2}$, Weikang Zhou ${ }^{2}$, Fan Feng ${ }^{2}$ and Ding Zhang ${ }^{2}$ \\ ${ }^{1}$ Beijing Institute of Technology Beijing, China \\ ${ }^{2}$ Tianjin University Tianjin, China
}

\begin{abstract}
In this paper, we present a new RDF engine accelerated by GPU, named TriAG, to query the RDF graph efficiently. Firstly, to improve the processing efficiency of SPARQL on RDF, new storage models of RDF systems is proposed. Then we use query decomposition to further reduce the query response time; at the same time, a cost model based on machine learning is used to determine the granularity of query decomposition. After this, we develop a MapReduce-based algorithm to join solutions of SPARQL subqueries in a parallel way. Finally, we implement TriAG and evaluate it by comparing it with two popular SPARQL query engines, namely, gStore and RDF3X on the LUBM benchmark. The experiments demonstrate that TriAG is highly efficient and effective.
\end{abstract}

Keywords: SPARQL,RDF,GPU

\section{Introduction}

The Resource Description Framework (RDF)[1] is a W3C Recommendation for the formulation of metadata on the World Wide Web. It provides a simple way to express facts in the semantic web and has been used in various applications. For example, UniProt, PubChemRDF, Bio2RDF, and DBpedia. These databases are usually interlinked and are globally queried using SPARQL[2].

Queries over RDF graphs can be expressed by SPARQL[2], which is the standard query language over RDF datasets. With the rapid growth of RDF data and the high complexity of SPARQL, processing SPARQL queries effectively becomes a significant challenge.

At an early phase, most solutions are proposed in a single machine, such as Jena [3], Sesame[4], RDF3X[5], Hexastore[6], and gStore. They employ various kinds of storage methods, optimizing strategies, and query approaches to improve the performance of query processing. However, they can not handle large-scale RDF data and answer complex queries efficiently enough.

Thus, the parallel processing system is a critical technology to handle this problem. As a novel parallel processing way, graphics processing units (GPUs) have been successfully used for general-purpose computing tasks. GPUs provide a higher level of parallel processing by containing hundreds to thousands of compute units [7]. A high-performance GPU owns the ability to execute multiple threads simultaneously, which ensures the feasibility of many applications to process a large amount of data [8].

Combining the performance of GPU over RDF data, Pan [9] assumed a shared memory architecture to parallel RDFS reasoning on massively parallel hardware. Despite the performance of RDFS reasoning improved, this method has not strong universality in RDF data processing. [10][11] presents a Jena-based RDF processing system by key-value structure and HDT-based binary triple pattern. TripleID [11] was proposed as an RDF parallel processing framework for query answering and reasoning. ${ }^{1}$

In this paper, we propose a new RDF engine, named TriAG to accelerate SPARQL query processing via GPU. The contributions of our work are summarized as follows:

${ }^{+}$Corresponding author.

E-mail address: shujunwang@tju.edu.cn. 
- We present a top-down query decomposition algorithm to split SPARQL queries into multiple SPARQL subqueries to reduce the scale of internal results. Moreover, we develop a MapReduce-based algorithm to join solutions of SPARQL subqueries for parallel processing of SPARQL queries.

- A new fast RDF organization structure, named TriStore in memory is proposed. It improves the execution speed of SPARQL on RDF.

- We implement TriAG and evaluate it by comparing it with two popular SPARQL query engines, namely, gStore and RDF3X on the LUBM benchmark[12]. The experiments demonstrate that TriAG is highly efficient and effective.

\section{RDF Engine}

\subsection{RDF Data Storage}

We discuss the commonly used RDF data management schemes firstly. Because the storage of RDF is of considerable significance to improve the execution efficiency of SPARQL.

Triple table only uses one table with three columns corresponding to subject, predicate, and object to store RDF data[13]. An index is created on each column to ensure faster join operations. But, this approach of data storage will lead to expensive self joins. Hexastore[14] reduces this cost by using a set of indices that cover all possible permutations of subject, predicate and object. Although this RDF management brings a significant improvement, it requires redundant storage of six pieces of data.

Property table[15] is a broader and flattened representation of RDF data. This representation can't represent multi-valued attributes. Vertical Partitioning is another representation of RDF data proposed by SW-Store[16]. The triples table is vertically partitioned into $\mathrm{n}$ tables, where $\mathrm{n}$ is the number of distinct predicates. This approach stores multi-valued attributes as successive rows and does not store NULL values. It provides excellent performance for queries with bounded predicates. However, it requires scanning multiple tables to reconstruct information related to a single entity. In this paper, we propose a novel approach to manage RDF data(named TriStore). Figure 1 is an example of a TriStore used to store RDF data. Figure 1(a) represents the original RDF data, to save memory usage. Instead of storing entire strings or URIs, we use shortened versions or keys. In particular, we map string URIs to integer identifiers. The encoding rule is (taking the encoding of the subject as an example, assuming that the current maximum encoding of the subject is $\mathrm{m}$, and the next string $\mathrm{s}$ has not appeared yet, then the encoding of the string $\mathrm{s}$ is $\mathrm{m}+1$ ). For each RDF element value, TriStore also maintains a mapping table that maps these keys to their corresponding strings. After encoding, we convert Figure 1(a) to Figure 1(b).

In order to match queries more efficiently, we use three tables(Figure 1(c), Figure 1(d), Figure 1(e)) to store set of triples Di in an in-memory data structure, which supports the following search operations, where $\mathrm{s}, \mathrm{p}$ and $\mathrm{o}$ are subject, predicate and object:

1. given $\mathrm{p}$, return set $\{(\mathrm{s}, \mathrm{o}) \mid<\mathrm{s}, \mathrm{p}, \mathrm{o}>\in \mathrm{Di}\}$

2. given $\mathrm{s}$ and $\mathrm{p}$, return set $\{\mathrm{o} \mid<\mathrm{s}, \mathrm{p}, \mathrm{o}>\in \mathrm{Di}\}$

\begin{tabular}{|c|c|c|}
\hline subject & predicate & object \\
\hline rdf:George & rdf:hasJob & $\begin{array}{c}\text { rdf:FullProfes } \\
\text { sor }\end{array}$ \\
\hline rdf:George & rdf:liveIn & $\begin{array}{c}\text { rdf:United } \\
\text { States }\end{array}$ \\
\hline $\begin{array}{c}\text { rdf:United } \\
\text { States }\end{array}$ & rdf:type & rdf:Country \\
\hline $\begin{array}{c}\text { rdf:United } \\
\text { States }\end{array}$ & rdf:liveIn & $\begin{array}{c}\text { rdf:United } \\
\text { States }\end{array}$ \\
\hline $\begin{array}{c}\text { rdf:FullProfes } \\
\text { sor }\end{array}$ & rdf:type & rdf:Professor \\
\hline rdf:Professor & rdf:workAt & rdf:University \\
\hline rdf:Canada & rdf:type & rdf:Country \\
\hline
\end{tabular}

(a)

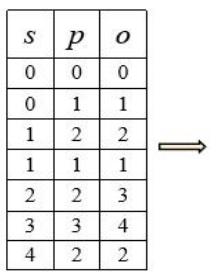

(b)

\begin{tabular}{|c|c|c|}
\hline $\begin{array}{c}\text { Row } \\
\text { number }\end{array}$ & PID & $($ SID, OID $)$ \\
\hline 0 & 0 & $(0,0)$ \\
\hline 1 & 1 & $(0,1)(1,1)$ \\
\hline 2 & 2 & $(1,2)(2,3)(4,2)$ \\
\hline 3 & 3 & $(3,4)$ \\
\hline
\end{tabular}

(c)

\begin{tabular}{|c|c|c|}
\hline $\begin{array}{c}\text { Row } \\
\text { number }\end{array}$ & SID & $<$ PID, OID $>$ \\
\hline 0 & 0 & $<0,0\rangle<1,1>$ \\
\hline 1 & 1 & $<2,2><1,1>$ \\
\hline 2 & 2 & $<2,3>$ \\
\hline 3 & 3 & $<3,4>$ \\
\hline 4 & 4 & $<2,2>$ \\
\hline
\end{tabular}

(d)

\begin{tabular}{|c|c|c|}
\hline $\begin{array}{c}\text { Row } \\
\text { number }\end{array}$ & OID & $\langle$ PID, SID $\rangle$ \\
\hline 0 & 0 & $<0,0\rangle$ \\
\hline 1 & 1 & $\langle 1,0\rangle\langle 1,1\rangle$ \\
\hline 2 & 2 & $\langle 2,1\rangle\langle 2,4\rangle$ \\
\hline 3 & 3 & $<2,2\rangle$ \\
\hline 4 & 4 & $<3,3\rangle$ \\
\hline
\end{tabular}

(e)

Fig.1:RDF Store

3. given $o$ and $p$, return set $\{s \mid<s, p, o>\in D i\}$ 
Where Figure 1(c), Figure 1(d), and Figure 1(e) are used to support query types 1, 2 and 3 above, respectively. For the first type of query, only the predicate $\mathrm{p}$ is known, and we want to get all p-related $\mathrm{s}$ and o. For triStore, we first get the encoding number $m$ of string $p$, and then we only need to output all the data of line $m$ in Figure 1(c) (it can be observed that the line number of the table is equal to the encoding of the predicate). For example, we perform this SPARQL query(Select* where\{?x rdf : type ?y.\}). First, find the digital encoding of the predicate rdf : type, after finding that the encoding is 2 , then directly output all the data in the second row of Figure 1(c). Therefore, after obtaining the encoding of string p, corresponding data can be quickly found with $\mathrm{O}(1)$ time complexity.

Figure 1(d) used to support fast matching of the second type of query, where $s$ and $p$ are known. What we want to get is $o$, which is related to both $s$ and $p$. For TriStore, the encoding $m$ and $n$ of string $s$ and $p$ should be obtained first. As we can see from the running examples, line $m$ in Figure 1(d) stores all PIDs and OIDs associated with the SID $m$ (there is a mapping table that maps PIDs to OIDs). Hence, we only need to output all the data in the m-th line with the key $\mathrm{n}$.

\subsection{Query Optimization}

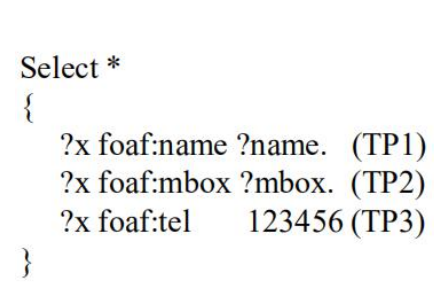

(a)

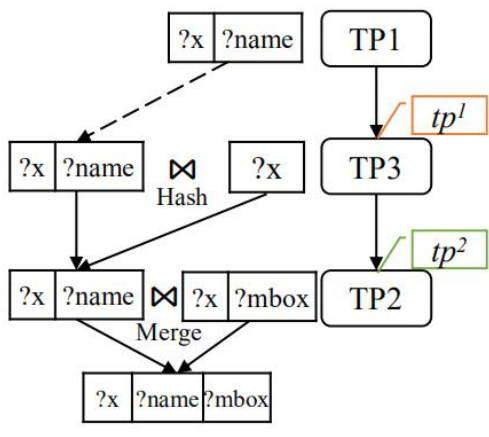

(b)

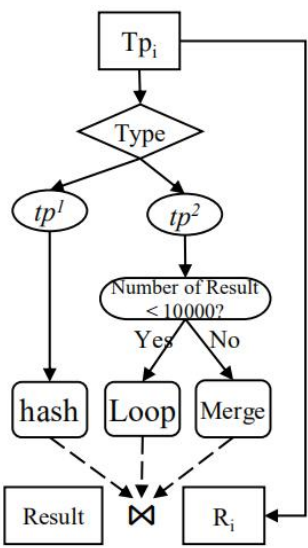

(c)

Fig.2: An example of the choice of Join method

The key issue for optimizing SPARQL execution plans is joining order. A basic SPARQL query consists of multiple subqueries(triple patterns): $\mathrm{q}_{1}, \mathrm{q}_{2}, \ldots, \mathrm{q}_{\mathrm{n}}$. If there are $\mathrm{n}$ triple patterns in a SPARQL query, the number of possible joining orders is $n$ !. Hence, we chose to use the dynamic programming (DP) method given in RDF3X[5] to generate the best joining order.

Another key issue for optimizing SPARQL execution plan is join method. There are three main types of join methods(Nested-Loop, MergeJoin and HashJoin). There are usually two types of triple patterns in SPARQL, one containing only one variable (denoted as tp ${ }^{1}$ ), such as TP1 and TP2 in Figure 2(a), and the other containing two variables (denoted as $\mathrm{tp}^{2}$ ), such as TP3 in Figure 2(a).

Figure 2(c) shows our strategy for choosing the join method. Where $R_{\mathrm{i}}$ is the result of TPi return. Assuming that there is already a partial result $R$, then we need to execute $R$ jion $R_{i}$. The choice of join method depends on the type of TPi. If TPi is of type tp ${ }^{1}$, then hashJoin is chosen (because the triple pattern of tp1 type is more restrictive and the number of $R_{i}$ is small). If TPi is of the $\mathrm{tp}^{2}$ type, the selection between mergeJoin and LoopJoin is made according to the number of rows in the result of $R_{i}$. If the number of results is less than 10,000, select LoopJoin.

Let's assume that (b) in Figure 2 is the best joining order for query (a). Figure 2(b) is an example of choosing the join method.

\section{TriAG for Parallel Processing SPARQL Queries}

\subsection{SPARQL Classifier}

Most of the current query optimization work is around BGP in SPARQL. A huge number of engines can only execute BGP-only SPARQL queries. To enable the query engine to support more types of SPARQL 
queries, we chose to use D2RQ to convert more than BGP-containing SPARQL into SQL and execute it on the relational database.

\subsection{Cost Model}

In this section, we address the problem of predicting SPARQL query performance. We define feature vectors, $\mathrm{x}=\left(\mathrm{x}_{1}, \mathrm{x}_{2}, \ldots \mathrm{x}_{\mathrm{n}}\right)$, where $\mathrm{xi}$ is a SPARQL query feature. The performance metric, query execution time, is the variable $\mathrm{y}$. We learn a function $\mathrm{f}(\mathrm{x})=\mathrm{y}$, i.e., the function maps a feature vector $\mathrm{x}$ to $\mathrm{y}$, using regression. In order to utilize machine learning algorithms for SPARQL query performance prediction, we transform the query into a vector representation.

SPARQL Algebra Features We use the frequencies of all the SPARQL algebra operators as query features. Besides, we also use these features: the depth of the algebra expression tree, the number of triple patterns and the number of results per triple pattern in the data set (given by statistics).

Graph Pattern Features The SPARQL algebra features do not represent graph patters appearing in SPARQL queries. Transforming graph patterns to vector space is not trivial because the space is infinite. To address this, we create a query pattern vector representation relative to the query patterns appearing in the training data. First, we cluster the structurally similar query patterns in the training data into $\mathrm{K}$ number of clusters. The query pattern in the center of a cluster is the representative of query patterns in that cluster. Second, we represent a query pattern as a $\mathrm{K}$ dimensional vector where the value of a dimension is the structural similarity between that query pattern and the corresponding cluster center query pattern.

In this work, we choose KNN regression algorithm to train the data set, Because k-NN is suitable for irregular data points.

Prediction Models k-NN is a non-parametric classification and regression method. The k-NN algorithm predicts based on the closest training data points. It uses a distance function to compute these closest data points. We use Euclidean distance as the distance function in our experiments. For predictions, we use the weighted average of the $\mathrm{k}$ nearest neighbors - weighted by the inverse of the distance from the querying data point. This ensures that the nearby neighbors contribute more to the prediction than the faraway neighbors.

$$
f(Q)=f(x)=y
$$

After training on the data set, we can get a function like Formula 2 to estimate the SPARQL cost. Suppose we have a SPARQL query q, and q can be decomposed into two subqueries q1 and q2. The cost before query decomposition is $\mathrm{f}(\mathrm{q})$, and the cost after decomposition is $\left(f\left(q_{1}\right)+f\left(q_{2}\right)\right)$, since the query is decomposed, parallel methods can be used to execute (such as multi-threaded and distributed). Then, $f\left(q_{1}\right)+$ $f\left(q_{2}\right)$ can be converted to $\operatorname{Max}\left\{f\left(q_{1}\right), f\left(q_{2}\right)\right\}$. Therefore, we believe that when $\operatorname{Max}\left\{f\left(q_{1}\right), f\left(q_{2}\right)\right\}<f(q)$, the query $\mathrm{q}$ can be decomposed, but in fact, after the results of $\mathrm{q}_{1}$ and $\mathrm{q}_{2}$ are obtained, we need to perform an intersection operation to obtain the final results(this operation will take some extra time.). Hence, the final judgment formula is $\operatorname{Max}\left\{f\left(q_{1}\right), f\left(q_{2}\right)\right\}+t_{j}<f(q)$. After testing, $t_{j}$ was set to $0.15 * f(q)$.

\section{Experiments and Datasets}

CPU vs. GPU In this experiment, we evaluate the GPU performance of TriAG by comparing it with CPU over different datasets, including LUBM20, LUBM40, LUBM60, LUBM80 and LUBM100. And all queries selected in this experiment can also be founded in [12](here we renumber them).

Table 1: Query Response Time of Q1 and Q2(ms)

\begin{tabular}{llll}
\hline & CPU & GPU & speedup \\
\hline LUBM20 & 826.4 & 270.86 & 3.05 \\
LUBM40 & 1725.57 & 524.12 & 3.29 \\
LUBM60 & 2781.56 & 808.58 & 3.44 \\
LUBM80 & 3607.96 & 1079.44 & 3.34 \\
LUBM100 & 4955.43 & 1371.61 & 3.61 \\
\hline
\end{tabular}

\begin{tabular}{llll}
\hline & CPU & GPU & speedup \\
\hline LUBM20 & 1660.88 & 608.44 & 2.72 \\
LUBM40 & 3466.52 & 1103.94 & 3.14 \\
LUBM60 & 5587.44 & 1599.62 & 3.49 \\
LUBM80 & 7248.28 & 2208.06 & 3.28 \\
LUBM100 & 9616.86 & 2616.41 & 3.67 \\
\hline
\end{tabular}


The experimental results are shown in Table 1 . The left table shows the query response time of $\mathrm{Q}_{1}$ and the right one shows the query response time of $\mathrm{Q}_{2}$. The Figure3 is the corresponding data graph of Table 1 .

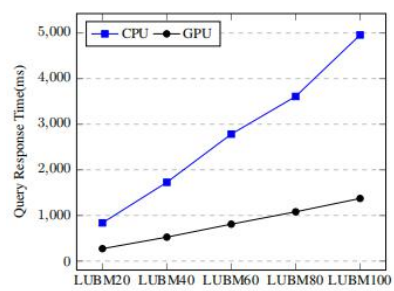

(a) Query Response Time of $\mathrm{Q}_{1}$

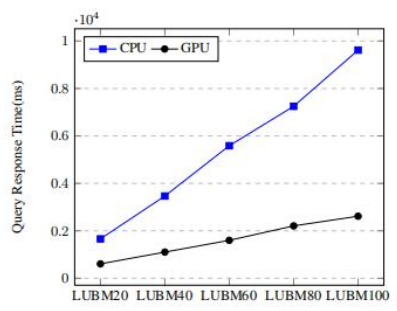

(c) Query Response Time of $\mathrm{Q}_{2}$

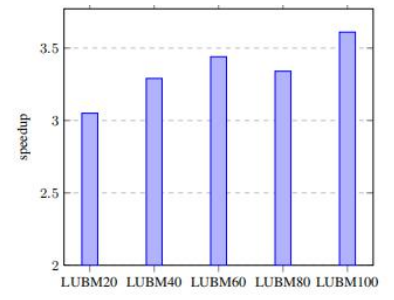

(b) speedup between CPU and GPU of $\mathrm{Q}_{1}$

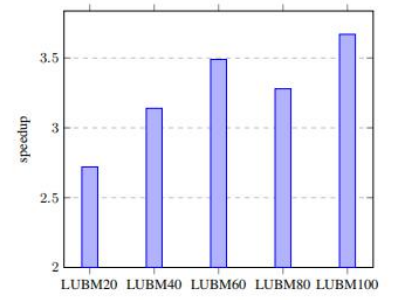

(d) speedup between CPU and GPU of $\mathrm{Q}_{2}$

Fig 3: CPU vs. GPU

Figure 3(a) shows the query response time of $\mathrm{Q}_{1}$ on different scale LUBM datasets. Figure 3(b) shows the speed-up ratio of CPU-based query response time and the GPU-based. Figure 3(c) shows the query response time and 9(d) shows the speed-up rate for $\mathrm{Q}_{2}$ on different scale LUBM datasets.

According to the experimental results, the query response time of TriAG is similar to the performance of the CPU-based implementation over less data. The reason is that the process of calling the GPU requires an additional cost of communication. However, with the increasing of data scale, query response time of GPU is far lower than the CPU due to GPU parallel computing power.

Figure 3(a) and Figure 3(c) show, GPU query response time significantly lower than the CPU. The speed-up ratio of CPU and GPU gradually increased, and finally stabilized at about three times. Compared with the experimental results, the efficiency of GPU is significantly higher than the CPU.

TriAG vs. SPARQL Query Engines To test and analyze the algorithm proposed in this paper, this experiment is designed to test different datasets of the same query and different queries of the same dataset. The goal of the experiment is confirming the efficiency and extendibility of TriAG. In this paper, we select the representative queries of the LUBM standard queries as the benchmark of this experiment and test the ten different datasets.

Figure 4 and Figure 5 show the query response time of gStore, RDF3X, and TriAG. In Figure 4, we test several queries from $q_{1}$ to $q_{7}$ over the LUBM100 dataset to compare the performance of TriAG with others. And, in Figure 5, we test the same query: $q_{1}$ over various datasets. It shows that TriAG processes the SPAQRL query more efficiently than other engines.

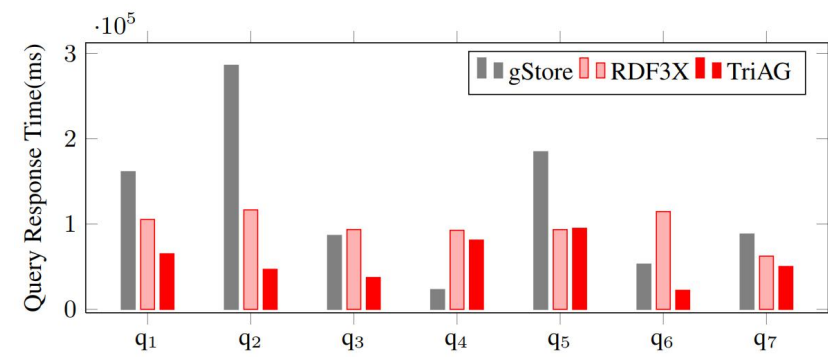

Fig. 4: Query Response Time of Different Queries $\mathrm{q}_{1}-\mathrm{q}_{7}$

The speedup of the above tests is shown in Table 2 where Table 2(a) is the speedup of Figure 4 and Table3(b) is the speedup of Figure 5. Combining the results of query response time and speedup, we can show that TriAG is more efficient than the performance of gStore and RDF3X. By comparing with gStore and RDF3X, TriAG can improve up to 6.10 in query response time. 


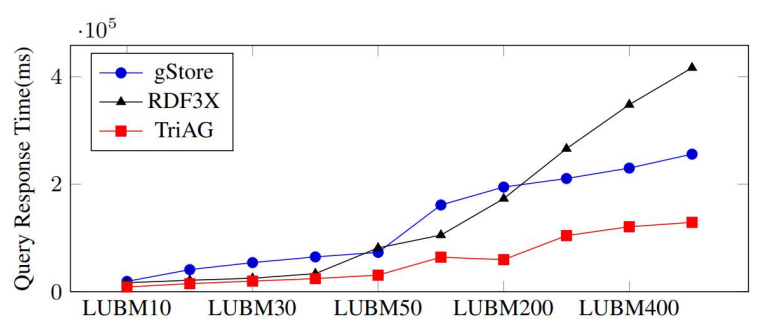

Fig.5: Query Response Time of Different Queries of Different Dataset

Table 2. Speedup of Query Response Time

\begin{tabular}{lll}
\hline & gStore & RDF3X \\
\hline $\mathrm{q}_{1}$ & 2.47 & 1.61 \\
$\mathrm{q}_{2}$ & 6.10 & 2.49 \\
$\mathrm{q}_{3}$ & 2.32 & 2.51 \\
$\mathrm{q}_{4}$ & 0.29 & 1.14 \\
$\mathrm{q}_{5}$ & 1.94 & 0.98 \\
$\mathrm{q}_{6}$ & 2.37 & 5.13 \\
$\mathrm{q}_{7}$ & 1.76 & 1.07 \\
\hline
\end{tabular}

\begin{tabular}{lll}
\hline dataset & gStore & RDF3X \\
\hline LUBM10 & 2.19 & 1.90 \\
LUBM20 & 2.74 & 1.42 \\
LUBM30 & 2.75 & 1.27 \\
LUBM40 & 2.66 & 1.38 \\
LUBM50 & 2.38 & 2.65 \\
LUBM100 & 2.52 & 1.64 \\
LUBM200 & 3.27 & 2.91 \\
LUBM300 & 2.02 & 2.55 \\
LUBM400 & 1.90 & 2.88 \\
LUBM500 & 1.98 & 3.23 \\
\hline
\end{tabular}

\section{(a)Speedup of Figure 4}

(b) Speedup of Figure 5

In Figure 4, the most query response time of TriAG is shorter than others, especially gStore and RDF3X. The reason why other engines faster is a particular structure of query is suitable for a certain engine. Figure 5 shows the great scalability of TriAG. TriAG is outstanding that its speedup rate is ranges from 1.3 to 6.1 in fewer datasets (from LUBM10 to LUBM50). And in large-scale datasets (from LUBM100 to LUBM500), the performance of TriAG is better than gStore and RDF3X. It still proves that TriAG shows good performance with increasing data volumes.

Experiment results show that our framework performs better than the RDF query engines and could efficiently speed up SPARQL queries answering.

\section{Conclusions}

In this paper, we proposed a new RDF Engine TriAG accelerated by GPU for parallel processing SPARQL queries. With this engine, we can take advantage of MapReduce and GPU in parallel computing so that large-scale RDF data can still be efficiently and effectively processed in TriAG. We believe that our proposal would be interesting to those researchers and engineers who are working on integrating parallel architecture with new hardware for processing SPARQL queries. Though we presented a smart strategy to split queries in our experiments, this strategy might not be optimal in handling other datasets, such as DBpedia. In the future works, We will study distributed versions of RDF Engine to support efficient SPARQL queries on larger RDF datasets.

\section{Acknowledgments}

This work is supported by Big Data Research Foundation of PICC.

\section{References}

[1] Hayes P. (2004)RDF Semantics: W3C Recommendation. 10 February 2004.

[2] Prud'hommeaux E. and Seaborne A. (2008) SPARQL query language for RDF. W3C Recommendation. 
[3] McBride B. (2001) Jena: Implementing the RDF model and syntax specification. In: Proc. of ISWC'01, pp.23--28.

[4] Broekstra J., Kampman A., and Van Harmelen F. (2002) Sesame: A generic architecture for storing and querying RDF and RDF schema. In: Proc. of ISWC'02, pp.54--68.

[5] Neumann T. and Weikum G. (2008) RDF-3X: A RISC-style engine for RDF. In: Proc. of VLDB'08, 1(1): 647-659.

[6] Weiss C., Karras P., Bernstein A. (2008) Hexastore: Sextuple indexing for semantic web data management. In: Proc. of VLDB'08, 1(1): 1008--1019.

[7] CUDA C best practices guide, v5. 5. NVIDIA, May, 2013.

[8] Zhang J., Zhang B., Zhang X., and Feng Z. (2016) IRSMG: Accelerating inexact RDF subgraph matching on the GPU. In: Proc. of ISWC'16, poster, CEUR Workshop Proceedings Vol. 1690.

[9] Heino N. and Pan J.Z. (2012) RDFS reasoning on massively parallel hardware. In: Proc. of ISWC'12, pp.133-148.

[10] Choksuchat C. and Chantrapornchai C. (2013) Experimental framework for searching large RDF on GPUs based on key-value storage. In: Proc. of JCSSE'13, pp.171--176.

[11] Choksuchat C. and Chantrapornchai C. (2013) On the HDT with the tree representation for large RDFs on GPU.In: Proc. of ICPADS'13, pp.651--656.

[12] Guo Y, Pan Z, Heflin J. (2005)LUBM: A benchmark for OWL knowledge base systems. J. Web Sem., 3(2): 158182.

[13] Ibrahim Abdelaziz, Razen Harbi, Zuhair Khayyat, and Panos Kalnis. A survey and experimental comparison of distributed sparql engines for very large rdf data. In: Proc. VLDB Endow., 10(13):2049--2060, September 2017.

[14] Cathrin Weiss, Panagiotis Karras, and Abraham Bernstein. Hexastore: Sextuple indexing for semantic web data management. Proc. VLDB Endow., 1(1):1008--1019, August 2008.

[15] K.Wilkinson.Jena Property Table Implementation In SSWS, pages 41--50,2006.

[16] Daniel J. Abadi, Adam Marcus, Samuel R. Madden, and Kate Hollenbach. Sw-store: a vertically partitioned dbms for semantic web data management. The VLDB Journal, 18(2):385--406, Apr 2009.

[17] Harris S., Lamb N., and Shadbolt N. (2009) 4store: The design and implementation of a clustered RDF store. In:Proc. of SSWS'09, pp.94--109.

[18] Perez J., Arenas M., and Gutierrez C. (2009) Semantics and complexity of SPARQL. ACM Trans. Database Syst., 34(3), article 16.

[19] Medha Atre, Vineet Chaoji, Mohammed J. Zaki, and James A. Hendler. Matrix "bit" loaded: A scalable lightweight join query processor for rdf data. In Proceedings of the 19th International Conference on World Wide Web, WWW '10, pages 41--50, New York, NY, USA, 2010. ACM.

[20] Kevin Wilkinson, Craig Sayers, Harumi Kuno, Dave Reynolds, and Jena Database. Efficient rdf storage and retrieval in jena2. Proceedings of SWDB, 022004.

[21] 2014 IEEE/WIC/ACM International Joint Conferences on Web Intelligence(WI) and Intelligent Agent Technologies (IAT), Warsaw, Poland, August 11-14, 2014 - Volume II. IEEE Computer Society, 2014.

[22] Mohammad Hammoud, Dania Abed Rabbou, Reza Nouri, Seyed-Mehdi-Reza Beheshti, and Sherif Sakr. DREAM: distributed $\{R D F\}$ engine with adaptive query planner and minimal communication.PVLDB, 8(6):654--665, 2015.

[23] Jiewen Huang, Daniel J. A Abadi, and Kun Ren. Scalable sparql querying of large rdf graphs. PVLDB, 4:1123-$1134,082011$.

[24] Kisung Lee and Ling Liu. Scaling queries over big rdf graphs with semantic hash partitioning. Proceedings of the VLDB Endowment, 6:1894--1905, 092013.

[25] Rohloff K. and Schantz R.E. (2010) High-performance, massively scalable distributed systems using the MapReduce software framework: The SHARD triple-store. In: Proc. of PSIETA'10, article 4.

[26] Saleem M. and Ngomo A.C.N. (2014) Hibiscus: Hypergraph-based source selection for SPARQL endpoint federation. In: Proc. of ESWC'14, pp.176--191. 